\title{
The Application of Latent Class Analysis for Diagnostic Test Validation of Chronic Trypanosoma cruzi Infection in Blood Donors
}

Dante M. Langhi Junior, José O. Bordin, Adauto Castelo, Stephen D. Walter, Hélio Moraes-Souza and Robert J. Stumpf

\author{
Hematology and Transfusion Medicine \\ Service, Santa Casa Medical School, São \\ Paulo; Hematology and Transfusion Medicine \\ Service, Federal University of São Paulo - \\ São Paulo School of Medicine, São Paulo; \\ Infectious Disease Service, Federal University \\ of São Paulo - São Paulo School of Medicine, \\ São Paulo, SP, Brazil; Department of Clinical \\ Epidemiology and Biostatistics, McMaster \\ University, ON, Canada; Hematology and \\ Transfusion Medicine Service, Federal \\ University of Uberaba, Minas Gerais, Brazil; \\ Abbott Diagnostics Division, Abbott \\ Laboratories, Abbott Park, IL, USA
}

\begin{abstract}
The main strategy to prevent transfusion-associated Chagas' disease is the identification of $T$. cruzi-infected blood donors by serological screening tests, however there is no perfect serological gold standard. We evaluated an enzyme immunoassay (EIA), an indirect hemaglutination (IHA), and an indirect immunofluorescence (IIF) test for detecting T. cruzi antibodies in Brazilian blood donors. The results were submitted to latent class analysis, and a radioimmunopreciptation (RIPA) test was performed on repeatedly positive samples. Among 1951 donors, $11(0.56 \%)$ were positive by EIA, $6(0.31 \%)$ by IHA and $16(0.82 \%)$ by IIF. Six samples were positive with all tests, while 4 reacted with EIA and IIF. The RIPA was positive in $6(75.0 \%), 7(66.6 \%)$, and $4(54.0 \%)$ samples reacting by the EIA, IHA and IIF tests, respectively. The latent class model detected a high sensitivity rate $(100 \%)$ for the EIA and IIF, and a specificity rate of $99.95 \%$ and $99.69 \%$ for the EIA and IIF tests, respectively. The probability of being case according to the model was $99.92 \%$ when both EIA and IIF were positive, and $100 \%$ for the association of EIA, IIF, and IHA.
\end{abstract}

Key Words: Chaga's disease, blood donors, diagnostic test, latent class analysis.

The current methods for detecting chronic T. cruzi infection are mainly based on identification of serum antibodies to the parasite [1] however, the serological diagnosis of infection is complex, falsely yielding both positive and negative results [2-4]. One additional

Received on 27 February 2002; revised 15 June 2002.

Address for correspondence: Dr. José O. Bordin. Universidade Federal de São Paulo, Hematology and Transfusion Medicine Service. Rua Botucatu, 740, São Paulo - SP, Brazil Zip code: 04023-902. Phone:(55-11)5574-0139. Fax (55-11)5571-8806. Email: jobordin@hemato.epm.br

The Brazilian Journal of Infectious Diseases 2002;6(4):181-187 (C) 2002 by The Brazilian Journal of Infectious Diseases and Contexto Publishing. All rights reserved. $1413-8670$ problem that these assays must overcome is a potential cross-reactivity with other related protozoan diseases, particularly leishmaniasis [5]. For situations such as this, where there is no perfect serological gold standard, latent class analysis may be used to evaluate the sensitivity and specificity of diagnostic tests [6]. This method assumes that the true disease state is not directly unobservable, but that the available methods of observation approximate the true state in some way $[7,8]$. We evaluated the performance of three commercially available serologic tests for detecting antibodies to T. cruzi among Brazilian blood donors. The serological results detected by enzyme immunoassay (EIA), indirect hemaglutination (IHA), 
and indirect immunofluorescence (IIF) methods were submitted to latent class model analysis. In addition, samples that gave repeatedly positive results by the serological screening tests were analyzed with a supplementary radioimmunopreciptation (RIPA) technique $[9,10]$.

\section{Materials and Methods}

Study population. The blood donor population consisted of 1951 subjects who donated blood in São Paulo City, a non-endemic area for Chagas' disease in Brazil. Since most of the donors originated from different regions of Brazil, they were selected by a pre-donation questionnaire that included questions regarding their epidemiological risk of $T$. cruzi infection. Donors were asked: (1) Do you know the insect that transmits Chagas' disease?; and/or (2) Have you ever lived in a house with contaminated with this insect?

Laboratory tests. All sera were screened by three commercially available tests, including: (1) second generation EIA that used purified antigens derived from epimastigote and amastigote forms of Y and CL T. cruzi strains (Abbott Laboratories, Abbott Park, IL) [11]; (2) IHA that employed antigens from epimastigote and amastigote forms of Y and CL T. cruzi strains at a 1-in-40 serum dilution (Biolab Diagnóstica, RJ, Brazil) [12]; and (3) IIF that was done using antigens from epimastigote forms of T. cruzi in a 1-to-20 serum dilution (Biolab Diagnóstica, RJ, Brazil) [13]. Results were considered positive or negative by the EIA according to the cut-off value obtained by the mean value of the optical density (OD) from the negative controls plus 0.360 , and then multiplied by the mean value of OD from the positive controls. An EIA result was considered negative at an OD identical to or higher than 0.020 but lower than the cut-off value, while an EIA result was considered initially reactive at an OD higher or identical to the cut-off value. The initially reactive samples were reevaluated in duplicate and if one of them was reactive, the sample was considered repeatedly reactive. If both duplicate tests were negative, the sample was considered negative for the presence of $T$. cruzi-antibodies.

For the IHA, results were considered negative when a pellet of red cells was formed at the bottom of the microplate well; results were considered positive when a uniform veil of red cells was formed covering all of the microplate well. A cloudy layer of red cells at the bottom of the microplate well was considered weakly positive or indeterminate. All initially positive or indeterminate samples were reevaluated in duplicate and if one of the duplicate tests was positive the sample was considered repeatedly positive.

A continuous emission of green fluorescence on the membrane of the T. cruzi in the IIF test was scored as a positive result while tests with an absent or a discontinuous (non-specific) pattern of green fluorescence emission on this membrane were considered negative. An IIF result was considered positive at a dilution titer of $\geq 20$, and IHA at a dilution titer of $\geq 40$.

To properly evaluate the sensitivity of the serological tests we examined 35 samples from persons with chronic $T$. cruzi infection confirmed by a xenodiagnosis test. The supplementary RIPA test was performed using a lysate prepared from extracted membrane proteins of $T$. cruzi. ${ }^{125} \mathrm{I}$ labeled proteins were incubated with each sample and then immunoprecipitated using Protein A Sepharose. The eluted proteins were electrophoresed using acrylamide gels and imaged using autoradiography film. Samples with antibodies to $T$. cruzi showed reactivity to $\mathrm{p} 32$ and $\mathrm{p} 34$, and a band at p90 gave additional supporting data $[9,10]$.

Statistical analysis. The latent class model allows for false-positive and false-negative errors to occur at certain rates in the test data; the test sensitivity is $1-$ FNR (false negative rate) and the test specificity is $1-$ FPR (false positive rate). For example, a positive test result is interpreted in the model as having come from either a true case of disease (thus, a correct result) or from a non-case (thus, an incorrect, false positive error). The mixing of these two possibilities occurs at unknown proportions, defined by the true disease prevalence [6]. 
Using data from a set of individuals with several test results each, the model can estimate the error rates (or, equivalency, sensitivity and specificity) for each test and the disease prevalence. Observational errors are assumed to be conditionally independent (i.e. given the true disease state of an individual) between tests. A minimum of three tests is required to fit the model without making further assumptions about the parameter values. Parameter estimation is achieved by standard maximum likelihood methods, which also yield standard errors for the parameters [6-8].

\section{Results}

Among 1,951 blood donors, 17 (0.87\%) showed positive results by at least one technique. Eleven $(0.56 \%)$ samples were positive by EIA, $6(0.31 \%)$ by IHA and $16(0.82 \%)$ by IIF. Only $6(0.31 \%)$ individuals gave positive results by all three tests, and $4(0.20 \%)$ samples reacted to only two tests (EIA and IIF). Four $(0.20 \%)$ individuals reacted exclusively to one test [3 $(0.15 \%)$ by IIF, and $1(0.05 \%)$ by EIA] (Table 1).

The results of the RIPA investigation are summarized in Table 2. The RIPA test was found to be positive in 6 $(85.7 \%)$ of $7,4(66.7 \%)$ of 6 and $7(58.3 \%)$ of 12 tested samples that reacted exclusively with the EIA, IHA and IIF tests, respectively. In addition, we found that the RIPA test was positive in $4(80 \%)$ of 5 samples that reacted simultaneously with the EIA, IHA and IIF tests, and in $2(100 \%)$ samples that were positive by EIA and IIF.

Table 3 shows the parameters estimated from the data incorporating EIA, IHA and IIF. All three tests appear to have excellent specificity values; the sensitivity was excellent for EIA and IIF, but IHA had a false negative rate of approximately 40 percent. Accordingly, the predictive values associated with almost all combinations of tests results were very close to 0 or 1 (Table 4).

The serological investigation of 35 known positive samples confirmed by a xenodiagnosis test showed a sensitivity rate of 100,100 and $88.2 \%$ for EIA, IIF, and IHA, respectively. The RIPA test was found to be positive in 29/31 (93\%), 29/31 (93\%), and 24/26 (92\%) positive samples confirmed by xenodiagnosis, and that were reactive by EIA, IIF, or IHA, respectively.

\section{Discussion}

The main strategies to prevent transfusion-associated Chagas' disease include the identification of putative infectious blood donors by risk history and by serological screening tests [1]. Since specific IgG levels rise soon after infection and titers of these antibodies remain high for life, the identification of T. cruzi-infected blood donors by serological tests has become the most accepted prophylactic alternative [14,15]. Typically, the sensitivity and specificity rates of the serological tests, which are available to detect antibodies to $T$. cruzi, are very high when sera from patients with infection confirmed by xenodiagnosis or from persons who are not exposed to T. cruzi infection are tested. However, there are many discrepancies among techniques when they are carried out to identify putative infectious blood donors [16].

We used 1951 sera obtained from Brazilian blood donors, without risk history for T. cruzi infection, for evaluating three serological assays routinely used for the detection of IgG antibodies against $T$. cruzi. When the results of three tests were submitted to a latent class model, we found not only a high sensitivity rate (100\%) for the EIA and IIF techniques, but also a high specificity rate of $99.95 \%$ and $99.69 \%$ for the EIA and IIF, respectively. Although, the estimated sensitivity rate for IHA was quite low $(60.02 \%)$, this test showed a high specificity rate (100\%). The probability of being case according to latent class model analysis was 99.92\% when both EIA and IIF tests were positive, and $100 \%$ for the association of the EIA, IIF, and IHA techniques.

Based on the estimated $100 \%$ sensitivity of EIA and IIF, if these tests are used in isolation there is still a possibility for a false positive result, since their estimated specificity is less than $100 \%$ (Table 3 ). However, based on the latent class model results, in all instances where 
Table 1. Results of the serological tests and the RIPA technique for the 17 blood donors samples repeatedly reactive for Trypanasoma cruzi antibodies

\begin{tabular}{|c|c|c|c|c|}
\hline Sample \# & $\begin{array}{c}\text { EIA } \\
\text { sample/cut-off ratio }\end{array}$ & IHA titer & IIF titer & RIPA \\
\hline 3397 & 1.5 & Negative & $1: 20$ & $\mathrm{p} 32+, \mathrm{p} 34+$ \\
\hline 7497 & 1.4 & Negative & Negative & Not done \\
\hline 71406 & 3.2 & Negative & $1: 20$ & Not done \\
\hline 71440 & 3.2 & $1: 1280$ & $1: 160$ & $\mathrm{p} 32+, \mathrm{p} 34+$ \\
\hline 2673 & 2.2 & Negative & $1: 40$ & Not done \\
\hline 448 & 3.4 & $1: 1280$ & $1: 80$ & $\mathrm{p} 32+, \mathrm{p} 34+$ \\
\hline 75254 & 1.5 & Negative & $1: 40$ & $\mathrm{p} 32+, \mathrm{p} 34+$ \\
\hline 75315 & 3.6 & $1: 80$ & $1: 160$ & $\mathrm{p} 32+, \mathrm{p} 34+$ \\
\hline 76224 & 2.9 & $1: 2560$ & $1: 160$ & $\mathrm{p} 32+, \mathrm{p} 34+$ \\
\hline 76233 & 3.2 & $1: 40$ & $1: 160$ & Not done \\
\hline 66611 & 3.6 & $1: 80$ & $1: 160$ & p32+ \\
\hline 47410 & Negative & Negative & $1: 40$ & Negative \\
\hline 48010 & Negative & Negative & $1: 20$ & $\mathrm{p} 32+, \mathrm{p} 34+$ \\
\hline 75337 & Negative & Negative & $1: 40$ & Not done \\
\hline 73311 & Negative & Negative & $1: 20$ & Negative \\
\hline 74211 & Negative & Negative & $1: 20$ & Negative \\
\hline 76411 & Negative & Negative & $1: 40$ & Negative \\
\hline
\end{tabular}

EIA, enzyme immunoassay; IHA, indirect hemaglutination; IIF, indirect immunofluorescence; RIPA, radioimmunoprecipitation assay.

Table 2. Results of the RIPA in samples repeatedly reactive by serological tests performed to identify chronically Trypanasoma cruzi-infected blood donors

\begin{tabular}{lccccc}
\hline & $\begin{array}{c}\text { Reactive } \\
\text { Serological test }\end{array}$ & \multicolumn{4}{c}{ RIPA } \\
\cline { 3 - 6 } & samples & Positive (\%) & Undetermined (\%) & Negative (\%) & Not done \\
\hline EIA & 11 & $6 / 7(85.7)$ & $1 / 7(14.2)$ & 0 & 4 \\
IHA & 6 & $4 / 6(66.7)$ & $1 / 6(16.6)$ & 0 & 1 \\
IIF & 16 & $7 / 12(58.3)$ & $1 / 12(8.3)$ & $4 / 12(33.3)$ & 4 \\
EIA, IHA and IIF & 6 & $4 / 5(80)$ & $1 / 5(20)$ & 0 & 1 \\
EIA and IIF & 4 & $2 / 2(100)$ & 0 & 0 & 2 \\
\hline
\end{tabular}


Table 3. Sensitivity and specificity rates for EIA, IHA and IIF tests performed to identify blood donors chronically infected with Trypanasoma cruzi

\begin{tabular}{lccc}
\hline \multicolumn{2}{c}{ Sensitivity } & \\
\hline \multicolumn{2}{c}{ Estimated Prevalence $=0.0051$} & $\mathrm{SE}=0.0016$ & $\begin{array}{c}95 \% \text { CI } \\
(0.002-0.008)\end{array}$ \\
\hline Test & Estimated Sensitivity (\%) & SE & $\mathbf{9 5 \%}$ CI \\
\hline EIA & 100 & 0.000 & $100-100$ \\
IHA & 60.02 & 0.155 & $29.6-90.4$ \\
IIF & 100 & 0.000 & $100-100$ \\
\hline & Specificity & & $95 \%$ CI \\
\hline Estimated Prevalence $=0.0051$ & SE $=0.0016$ & $(0.002-0.008)$ \\
\hline Test & Estimated Specificity (\%) & & $\mathbf{9 5 \%}$ CI \\
\hline EIA & 99.95 & SE & $99.8-100$ \\
IHA & 100 & 0.000 & $100-100$ \\
IIF & 99.69 & 0.000 & $99.4-100$ \\
\hline
\end{tabular}

EIA, second generation enzyme immunoassay; IHA, indirect hemaglutination; IIF, indirect immunofluorescence.

Table 4. Probability of being a Trypanasoma cruzi-infected blood donor according to the results of the EIA, IHA and IIF tests

\begin{tabular}{cccc}
\hline EIA-A & IHA-B & $\begin{array}{c}\text { Probability of } \\
\text { IIF-B }\end{array}$ & Being Case (\%) \\
\hline 0 & 0 & 0 & 0 \\
1 & 0 & 0 & 0 \\
0 & 1 & 0 & 0 \\
1 & 1 & 0 & 85.71 \\
0 & 0 & 1 & 0 \\
1 & 0 & 1 & 99.92 \\
0 & 1 & 1 & 50.00 \\
1 & 1 & 1 & 100 \\
\hline $0=$ Negative; 1 = Positive; EIA, second generation enzyme immunoassay; IHA, indirect \\
hemaglutination; IIF, indirect immunofluorescence.
\end{tabular}


one of the two tests (EIA or IIF) was positive and the other one negative, it was always considered a false positive result (negative predictive value, NPV) [Table $3]$. In contrast, when both EIA and IIF are positive there is $99.92 \%$ confidence that the result comes from a true positive case (positive predictive value, PPV) [Table 3]. This very high PPV can be further increased to $100 \%$ if the IHA test is also positive (Table 3 ).

The RIPA has been used to screen sera for anti- $T$. cruzi IgG antibodies and to classify a sample as confirmed seroreactive, indeterminate, or nonreactive. Recently, a combination of two confirmatory assays, ELISA and RIPA, has been reported to result in a sensitivity and specificity approaching $100 \%[9,10]$. The results observed with RIPA in our study are more or less in accordance with what was demonstrated by the latent class model, suggesting that when both EIA and IIF are positive, the confidence that the result comes from a true positive case (PPV) is near $100 \%$ (Table 4). However, RIPA is a very time-consuming technique, the number of samples that can be analyzed at a time is quite limited, and a more suitable technique is still needed to confirm positive results found with the serological screening assays routinely used in blood banks to detect antibodies to T. cruzi.

Some countries have required that two tests be performed for blood bank screening of Chagas' disease because substantial uncertainty surrounds the sensitivity and specificity of the serological tests for $T$. cruzi infection in blood donors, and no good gold standard exists for unequivocally identifying infected subjects. We explored the latent class analysis modeling technique to circumvent this problem. According to our results in this study, using latent class analysis, the strategy of using two different serological techniques seems to optimize test results as one can considerably increase the PPV of being case going from one to two tests. In contrast, not much is gained by adding a third test if the first two tests are EIA and IFF (Table 4). In conclusion, in the absence of a universally accepted gold standard to confirm positive test results for detecting $T$. cruzi-infected blood donors, by using the latent class model, the most appropriate strategy seems to be the association of EIA and IIF.

\section{Abbreviations}

T. cruzi $=$ Trypanossoma cruzi; TA-CD = transfusion-associated Chagas' disease; EIA = enzyme immunoassay; IHA = indirect hemaglutination; IIF = indirect immunofluorescence; $\mathrm{OD}=$ optical density; $\mathrm{FNR}$ $=$ false negative rate; $\mathrm{FPR}=$ false positive rate; $\mathrm{NPV}=$ negative predictive value; $\mathrm{PPV}=$ positive predictive value .

\section{References}

1. Moraes-Souza H., Bordin J.O. Strategies for prevention of transfusion-associated Chagas' disease. Transfusion Medicine Reviews 1996;10:161-70.

2. Chiari E. Diagnostic tests for Chagas disease. In: Wendel S., Brener Z., Camargo M.E., Rassi, A. [eds]. Chagas Disease (American Trypanosomiasis): Its Impact on Transfusion and Clinical Medicine. São Paulo: SBHH, 1992.

3. Camargo M. An appraisal of Chagas disease serodiagnosis. In: Wendel S., Brener Z., Camargo M.E., Rassi A. [eds]. Chagas Disease (American Trypanosomiasis): Its Impact on Transfusion and Clinical Medicine. São Paulo: SBHH, 1992.

4. Ferreira A.W. Tests for Chagas Disease serodiagnosis: a review. In: Wendel S., Brener Z., Camargo M.E., Rassi A. [eds]. Chagas Disease (American Trypanosomiasis): Its Impact on Transfusion and Clinical Medicine. São Paulo: SBHH, 1992.

5. Camargo M.E., Segura E.L., Kagan I.G., et al. Three years of collaboration on the standardization of Chagas' disease serodiagnosis in the Americas: an appraisal. Bulletin of the Pan American Health Organization 1986;20:233-44.

6. Goetghebeur E., Liinev J., Boelaert M., Van der Stuyft P. Diagnostic test analyses in search of their gold standard: latent class analyses with random effects. Statistical Methods and Medical Research 2000;9:231-48.

7. Szatmari P., Volkmar F., Walter S. Evaluation of diagnostic criteria for autism using latent class models. Journal of the American Academy of Child and Adolescence Psychiatry 1995;34:216-22.

8. Boelaert M., el Safi S., Goetghebeur E., et al. Latent class analysis permits unbiased estimates of the validity of DAT for the diagnosis of visceral leishmaniasis. Tropical Medicine and International Health 1999;4:395-401.

9. Brashear R.J., Winkler M.A., Schur J.D., et al. Detection of antibodies to Trypanosoma Cruzi among blood donors in the southwestern and western United States. Evaluation of the sensitivity and specificity of an enzyme immunoassay for detecting antibodies to $T$. cruzi. Transfusion 1995;35:213-8. 
10. Wrinkler M.A., Brashear R.J., Hall H.J., et al. Detection of antibodies to Trypanosoma cruzi among blood donors in the southwestern and western United States. II. Evaluation of a supplemental enzyme immunoassay and radioimmunoprecipitation assay for confirmation of seroreactivity. Transfusion 1995;35:219-25.

11. Pan A.A., Rosemberg G.B., Hurley M.K., et al. Clinical evaluation of na EIA for the sensitive and specific detection of serum antibody to Trypanosoma cruzi (Chagas disease). J Infect Dis 1992;165:585-8.

12. Camargo M.E., Hoshino-Shimuzo S., Siqueira G. Hemaglutination with preserved sensitized cells. A practical test for routine serologic diagnosis of American Trypanosomiasis. Rev Inst Med Trop S Paulo 1973; $15: 81-5$.

13. Camargo M.E. Fluorescent antibody test for the serodiagnosis of American Trypanosomiasis. Technical modification employing culture forms of Trypanosoma cruzi in a slide test. Rev Inst Med Trop S Paulo 1966;8:227-34.

14. Tanowitz H.B., Kirchhoff L.V., Simon D. (1992) Chagas' disease. Clinical and Microbiological Reviews 1992;5:400-19.

15. Israelski D.M., Sadler R., Araujo F.G., et al. Antibody response and antigen recognition in human infection with Trypanosoma cruzi. American Journal of Tropical Medicine and Hygiene 1988; 39:445-55.

16. Carvalho M.R., Krieger MA., Almeida E., et al. Chagas' disease diagnosis: evaluation of several tests in blood bank screening. Transfusion 1993;33:830-4. 